
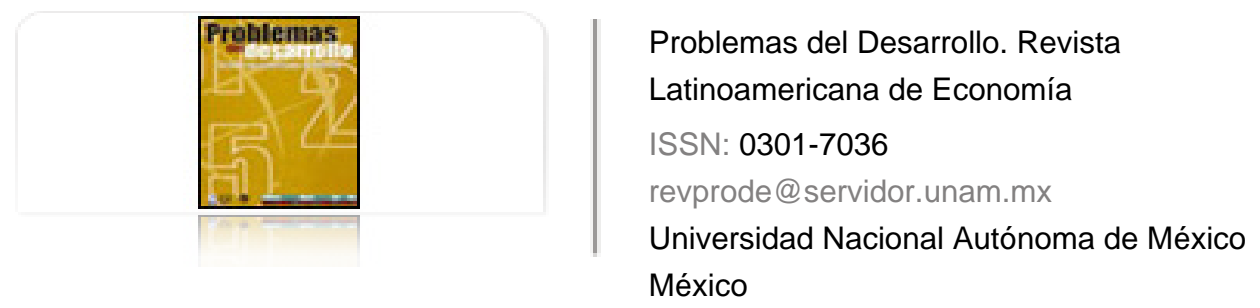

Barbosa Cano, Fabio

Situación de los campos petroleros en aguas profundas del mundo Problemas del Desarrollo. Revista Latinoamericana de Economía, vol. 39, núm. 155, octubrediciembre, 2008, pp. 101-125

Universidad Nacional Autónoma de México

Distrito Federal, México

Disponible en: http://www.redalyc.org/articulo.oa?id=11820123006

- Cómo citar el artículo

- Número completo

- Más información del artículo

Página de la revista en redalyc.org

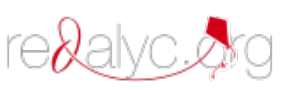

Sistema de Información Científica

Red de Revistas Científicas de América Latina, el Caribe, España y Portugal Proyecto académico sin fines de lucro, desarrollado bajo la iniciativa de acceso abierto 


\title{
SITUACIÓN DE LOS CAMPOS PETROLEROS EN AGUAS PROFUNDAS DEL MUNDO
}

\author{
Fabio Barbosa Cano*
}

Fecha de recepción: 22 de junio de 2008. Fecha de aceptación: 4 de septiembre de 2008.

\section{Resumen}

Este trabajo presenta un panorama de los campos en aguas profundas en el mundo, sobre todo los que se encuentran en operación. Intenta demostrar que la explotación en mares profundos es un fenómeno paralelo al actual ascenso de precios, cuya escalada es un asunto específico que requiere un análisis especial. No sabemos en qué medida intervienen en él la situación política y los problemas de oferta que sin duda están presentes empujando la exploración y extracción hacía áreas cada vez más difíciles. El artículo indaga sobre las innovaciones tecnológicas tendentes a acortar el ciclo entre el descubrimiento y la puesta en operación de los campos profundos, además sostiene que algunas de ellas se aplicarán a los campos en México con las ominosas consecuencias de precipitar la producción mexicana.

Palabras clave: hidrocarburos, exploración, explotación, aguas profundas y tecnología.

\section{Summary}

The present study presents a panorama of the world's deep-sea oilfields, especially those that are in operation. It aims to show that deep-sea exploitation is a phenomenon parallel to the current rise in prices, whose escalation is a specific affair requiring special analysis. We do not know to what extent they are affected by the political situation and supply problems that are undoubtedly present, pushing exploration and extraction towards increasingly more difficult areas. The article inquires into the technological innovations that tend to reduce the cycle between discovery and the coming into operation of deep-sea oilfields, and argues that some of them will apply to oilfields in Mexico with the ominous consequences of plummeting Mexican production.

Key words: hydrocarbons, exploration, exploitation, deep-sea and technology.

* Investigador asociado C de tiempo completo del Instituto de Investigaciones Económicas de la Universidad Nacional Autónoma de México. Correo electrónico: barbosa@servidor.unam.mx. 


\section{Résumé}

Ce travail présente un panorama des champs pétrolifères en eaux profondes dans le monde, essentiellement ceux qui sont en exploitation. Il tente de démontrer que l'exploitation en eaux profondes est un phénomène corrélatif à l'actuelle augmentation des prix, dont l'escalade est un sujet particulier qui requière une analyse spéciale. Nous ne savons pas dans quelle mesure la situation politique et les problèmes d'offre, dont l'existence ne fait aucun doute, y jouent un rôle, en poussant à explorer et extraire dans des endroits de plus en plus difficiles d'accès. L'auteur de l'article se livre à une enquête sur les innovations technologiques qui tendent à réduire le temps de passage de la découverte à la mise en exploitation des champs pétrolifères en eaux profondes, et soutient de plus que certaines d'entre elles seront appliquées aux champs pétrolifères des eaux territoriales du Mexique ayant pour conséquence abominable de précipiter la production mexicaine.

Mots clés: hydrocarbures, exploration, exploitation, eaux profondes et technologie.

\section{Resumo}

Este trabalho apresenta um panorama dos campos em águas profundas no mundo sobre tudo aqueles que se encontram em operação. Tenta demonstrar que a exploração em mares profundos é um fenômeno paralelo ao atual incremento de preços, cuja escalada é um assunto específico que precisa de uma análise especial. Desconhecemos a medida na que interferem nele tanto a situação política quanto os problemas de oferta que, induvidavelmente estão presentes conduzindo à exploração e extração para áreas cada vez mais difíceis. $O$ artigo indaga sobre as inovações tecnológicas tendentes a reduzirem o ciclo entre o descobrimento e o começo das operações dos campos profundos, além do mais diz que algumas delas serão aplicadas nos campos no México com as ominosas conseqüências da precipitação da produção mexicana.

Palavras chave: hidrocarbonetos, exploração, sondagem, águas profundas e tecnologia. 


\section{Introducción}

$\varepsilon$ n este artículo se ofrece una breve exposición de los campos en aguas profundas en el mundo. Sin pretender cubrir todos los aspectos de este segmento de la industria petrolera, investiga sobre las nuevas cuencas en exploración y en especial sobre los campos en operación. Nuestra hipótesis es que las perforaciones - en particular la explotación- en mares profundos han cobrado un impulso tan considerable en el actual ascenso de precios que podría postularse que son fenómenos concomitantes.

Las primeras incursiones en mares profundos datan de los años ochenta, pero los problemas de oferta en el mercado mundial han renovado el interés por esas áreas, al sugerir que los avances iniciales constituyeron hace más de 15 o 20 años sólo una etapa experimental y tentativa, en espera de los aumentos de precios que permitirían la definitiva extensión y generalización de las perforaciones profundas.

Uno de los objetivos de nuestra pesquisa es identificar por lo menos algunas de las innovaciones que se realizan en el curso del actual auge, sobre todo los orientados a acortar el ciclo entre el descubrimiento y la puesta en operación de los campos profundos. Algunos avances tecnológicos se aplicarán a los campos en México, con las ominosas consecuencias de precipitar la producción mexicana.

En este segmento de la industria petrolera, la pertinencia por conocer la situación internacional ayuda a comprender las presiones externas y los grupos que desde el interior del país pugnan por continuar el tipo de explotación orientada a las exportaciones y acelerar la incursión de Petróleos Mexicanos (PEMEX) hacia las aguas profundas del Golfo de México. Presentamos este trabajo cuando en el país se discute sobre la reforma energética y pretendemos que contribuya a un debate mejor informado.

La investigación se presenta en 21 cuadros estadísticos con la lista de los campos descubiertos y aquéllos en desarrollo en aguas profundas en el mundo. En el caso de Estados Unidos (EU), eliminamos la información desglosada, pues en ese país existe más de la mitad de los campos profundos en el mundo; incluir los detalles duplicaría la extensión de nuestro texto, de ahí que para esa nación se resume la información. Por otro lado, EU es un caso especial en la industria petrolera y su estudio puede realizarse por separado. ${ }^{1}$

1 Estados Unidos alcanzó el pico de su producción (peack oil) desde los años setenta, de ahí que inició inevitablemente desde entonces un avance a sus campos marinos profundos; a la fecha posee más de la mitad de este tipo de yacimientos. Por otro lado, el Golfo de México presenta condiciones muy difíciles que obligan a las petroleras a realizar experiencias que de 


\section{Algunas consideraciones sobre los criterios en la búsqueda de información}

En la industria petrolera no existe una convención internacional para definir qué debe entenderse por campos profundos. En Estados Unidos, país en donde se iniciaron estas actividades, se denomina aguas profundas a las situadas en tirantes de mil pies, es decir unos 300 metros. PEMEX y el Instituto Mexicano del Petróleo (IMP) se alinearon a esa definición durante décadas, hasta el sexenio pasado en el que empezaron a denominarse profundos sólo a los de más de 500 metros.

Luego de eludir el asunto de las definiciones, en nuestros cuadros estadísticos de este texto se incluyeron sólo los campos de más de 500 metros de profundidad de agua.

Los indicadores que se persiguieron en la búsqueda fueron los siguientes: 1) el nombre del campo, 2) la fecha de su descubrimiento; 3) la fecha del inicio de operación, que para nuestros objetivos es un dato fundamental; 4) la profundidad de agua, dato que por sí solo implica importantes desafíos operacionales; 5) el sistema de explotación instalado, un problema técnico sobre el que se están realizando algunas innovaciones que de seguro serán aplicadas en México, y 6) el nombre actual de la compañía operadora.

El dato sobre la producción (más propiamente extracción) es muy importante, pero no siempre puede tenerse acceso a las cifras desagregadas por campo; por otro lado, como adelante mostraremos, sólo en 14 países existen campos en operación, algunos apenas comenzaron en 2007 o en 2008, de ahí que incorporamos esas cifras sólo cuando se pudieron obtener.

Las cifras de las reservas probadas u otras estimaciones acerca del potencial siempre han sido objeto de interminables cuestionamientos. Cuando se examinan los cuadros estadísticos sobre las reservas en el mundo, la conclusión es que, con pocas excepciones, los descubrimientos y el inicio de operación de campos profundos no han repercutido, por lo menos hasta hoy, en la estadística mundial; es decir, que los incrementos en reservas probadas de las aguas profundas han sido poco significativos. Pero hay excepciones. En dos o tres países de la costa occidental de

modo alguno se han generalizado a otros países; por ejemplo sólo en EU se ha llevado a cabo una perforación más allá de 3,000 metros (por cierto resultó hoyo seco). Asimismo, es el único país del mundo donde la tecnología de explotación desarrollada le permite explotar campos en 2,400 metros de profundidad. El seguimiento que haremos mostrará que en Brasil el campo más profundo en producción se encuentra a 1,800 metros de profundidad, y en otros países los pozos de 2000 metros son verdaderas excepciones. 
África, un campo en Malasia otro en Noruega, es indudable que las aguas profundas han ofrecido descubrimientos importantes, incluso de campos gigantes (más de 500 millones de barriles) y sorprendentemente de supergigantes (más de 5000 millones de barriles), como parecen ser los recientemente descubiertos en Brasil. Por el interés de lo anterior, en la situación actual de dificultades de oferta en esos casos anotaremos los datos respectivos.

El recuento comprendió el universo completo: nueve cuencas: el Golfo de México, Brasil y la cuenca del Delta del Niger en la costa del occidente africano, el litoral occidental de Australia, el sur del Mar de China, los litorales de Japón y de India; el Mediterráneo y el margen del Atlántico frente a Noruega y norte de Escocia. Los campos están distribuidos en 22 países, incluyendo México. Los enumeramos a partir de la costa del occidente de África, en donde se encuentra la mayor concentración, inmediatamente después de EU.

África

Nigeria

En este país las primeras licitaciones de bloques en aguas profundas se realizaron desde 1990 y los primeros campos profundos se descubrieron en 1996, pero todos los campos descubiertos estuvieron inactivos, sin producir hasta que los precios iniciaron el actual ascenso en 2003. El siguiente cuadro muestra que todos los campos en explotación en ese país iniciaron operaciones precisamente en el periodo 2003-2008.

Quizá el gobierno de Nigeria se impacientó por no recibir los ingresos que le correspondían por la explotación petrolera, pues en 1998, 2000 y 2005 revocó las concesiones otorgadas a las empresas extranjeras en diversos bloques, para volver a licitarlas a otras que, suponemos, prometieran acelerar la explotación. ${ }^{2}$ El Departamento de Energía de Estados Unidos (DOE, por sus siglas en inglés) ha explicado que aunque la exploración y las evaluaciones comenzaron tempranamente desde los años noventa y se realizaron varios descubrimientos, la explotación ha sufrido demoras por los altos costos asociados con la explotación en aguas profundas. ${ }^{3}$

2 US, Department of Energy, "Energy profile of West Africa", publicado por la Energy Information Administration (www.eia.doe.gov).

3 "Exploration and appraisal began in 1995 and several discoveries have been made in Nigeria's deepwater although high costs associated with deep water have meant that production has been delayed", tomado de "Nigeria oil and gas: crude petroleum and natural gas extraction. Overview" (www.eia.doe.gov/emev/cabs/ECOWAS/pdf.pdf). 
Las empresas operadoras han puesto en actividad cuatro campos, uno de ellos, Bonga de Shell, es un yacimiento "gigante", así lo reportan consultorías petroleras y publicaciones especializadas ${ }^{4}$ y parece confirmarlo el volumen sobre la producción: 200 mil barriles de crudo diarios. Otro campo gigante en Nigeria es Usan de Chevron, cuyo arranque de operaciones está previsto para $2010 .{ }^{5}$

Cuadro 1

Campos de Nigeria

\begin{tabular}{|c|c|c|c|c|c|c|}
\hline Campo & $\begin{array}{c}\text { Fecha } \\
\text { de descubrimiento }\end{array}$ & $\begin{array}{l}\text { Fecha de inicio } \\
\text { de explotación }\end{array}$ & Tirante en metros & Compañía & Tipo de sistema & Situación actual \\
\hline 1.Abo & 1996 & 2003 & 580 & Agip (Italia) & $F P^{*}$ & En producción \\
\hline 2. Bonga Main & 1996 & 2005 & 1,030 & Shell & FP & En producción \\
\hline 3. Bosi & 1999 & $-\cdots+-$. & 1,458 & Exxon & -..-.- & Virgen \\
\hline 4. Erha & 1999 & 2006 & 1,036 & Exxon & FP & En producción \\
\hline 5. Akpo & 2000 & $\cdots+.$. & 1,325 & Total & .......... & Virgen \\
\hline 6. Ungo & 2002 & $-\cdots--\cdot$ & 700 & Exxon & -..--.-.- & Virgen \\
\hline 7. Usan & 2002 & -..---.- & 750 & Chevron & -..--..- & Virgen \\
\hline 8. Bonga Northwest & 2003 & -.-- & 1,200 & Shell & -...-.-.. & Virgen \\
\hline 9. Erha North & 2004 & 2006 & 1,000 & Exxon & SS to FP** & En producción \\
\hline 10. Eti/Asasa & $2005 ?$ & $-\cdots-\cdot-$ & ¿? & Chevron & -.---.- & Virgen \\
\hline 11. Agbami & $2006 ?$ & --.--.-- & ¿? & Chevron & -..--.-. & Virgen \\
\hline
\end{tabular}

Fuente: Para los campos descubiertos entre 1996-2004: Shell, boletines de prensa; Esso Exploration and Production Nigeria Limited, Petroleum Economist y Offshore, diversos números.

Los campos descubiertos entre 2005 y 2006 con frecuencia son citados en textos del DOE/EIA, pero no hemos podido completar nuestros datos.

*FP: Floating Production.

** SS to FP: Subsea Satellite to Floating Production.

\section{Angola}

Igual que en Nigeria, sus primeros campos profundos fueron descubiertos desde los años noventa, uno de ellos inició operaciones en 2002, pero todos los demás se han desarrollado hasta el periodo 2003-2008, es decir, el actual momento de alza de precios.

4 Douglas-Westwood Ltd\&Energyfiles, "The World Offshore Oil \& Gas Production \& Spend Forecast 2007-2011", citado en Adrian Jonh \& Georgie MacFarlan Douglas-Westwood Ltd, “Out of Africa. Deepwater Prospects $\$ 24.6 \mathrm{~B}$ by 2012", Maritime Reporter \& Engineering News, November 2007.

5 Chevron ha anunciado una producción de 180,000 barriles diarios, véase "Chevron confirms Development of Nigeria Deepwater field", Chevron Press Release (http://www.chevron.com). 
Angola parecería el país donde las grandes petroleras han desarrollado los mejores y más numerosos proyectos. Acaso esas grandes empresas han encontrado condiciones políticas menos desfavorables que en Nigeria y, tal vez, la geología es más

Cuadro 2

Campos profundos de Angola

\begin{tabular}{|c|c|c|c|c|c|c|}
\hline Campo & $\begin{array}{c}\text { Fecha } \\
\text { de descubrimiento }\end{array}$ & $\begin{array}{l}\text { Fecha de inicio } \\
\text { de explotación }\end{array}$ & Tirante en metros & Compañía & Tipo de sistema & Situación actual \\
\hline 1. Girasol & 1996 & 2001 & 1,360 & Total & FP & En producción \\
\hline 2. Kissanje & 1997 & 2005 & 1,011 & Exxon & FP & En producción \\
\hline 3. Dalia & 1997 & 2006 & 1,360 & Total & FP & En producción \\
\hline 4. Dalia II & 1998 & $-\cdots--$ & 1,250 & Total & -..----- & Virgen \\
\hline 5. Dikanza & 1998 & 2005 & 1,154 & Exxon & SS to FP & En producción \\
\hline 6. Hungo & 1998 & 2004 & 1,202 & Exxon & FP & En producción \\
\hline 7. Marimba & 1998 & $-\cdots---$ & 1,289 & Exxon & -..---- & Virgen \\
\hline 8. Rosa & 1998 & 2007 & 1,405 & Total & SS to FP & En producción \\
\hline 9. Chocalho & 1999 & 2004 & 1,147 & Exxon & ¿? & En producción \\
\hline 10. Xicomba & 1999 & 2003 & 1,355 & Exxon & FP & En producción \\
\hline 11. Camelia & 1999 & ----- & 1,296 & Total & -..---- & Virgen \\
\hline 12. Plutonio & 1999 & 2008 & 1,362 & BP o Exxon? & ¿? & En producción \\
\hline 13. Cravo & 1999 & -..-- & 1,357 & Total & -..---.- & Virgen \\
\hline 14. Cobalto & 2000 & -.-.-- & 1,330 & bp & -..--.-.- & virgen \\
\hline 15. Girasol C-1, Block 17 & 2000 & 2003 & 1,292 & total & SS to FP & En producción \\
\hline 16. Cromio & 2000 & $-\cdots---$ & 1,233 & BP & -..----- & Virgen \\
\hline 17. Galio & 2000 & $-\cdots---$ & 1,238 & BP & -..---.- & Virgen \\
\hline 18. Paladio & 2000 & -..--- & 1,230 & BP & -..---.- & Virgen \\
\hline 19.Tomboco & 2000 & -..--. & 506 & Gulf-cabinda & -..---.- & Virgen \\
\hline 20. Batuque & 2000 & -..-- & 725 & Exxon & -.-----. & Virgen \\
\hline 21. Mondo & 2000 & --.-- & 740 & Exxon & -..----.- & Virgen \\
\hline 22. Saxi & 2000 & $-\cdots---$ & 675 & Exxon & -..---.- & Virgen \\
\hline 23. Plutao & 2002 & -..--. & 2,020 & BP & -..---.- & Virgen \\
\hline 24. Saturno & 2003 & $-\cdots---$ & 1,804 & BP & -..---.- & Virgen \\
\hline 25. Gimboa & 2004 & $-\cdots--$ & 711 & Sonangol* & -.----.-. & Virgen \\
\hline 26. Bavuca & 2004 & $-\cdots--$ & 1,094 & Exxon & ------.- & Virgen \\
\hline 27. Marte & 2004 & .....- & 2,000 & BP & $\cdots \cdots+\cdots$ & Virgen \\
\hline 28. Venus & 2004 & -..--- & 2,012 & BP & -..---.. & Virgen \\
\hline 29. Cordelia & 2007 & -..--- & 2,308 & BP & -..---.- & Virgen \\
\hline 30.Cominhos & 2007 & -.--- & 1,594 & Total & -..-- & Virgen \\
\hline
\end{tabular}

*Sociedade Nacional de Combustiveis de Angola.

Fuente: Petroleum Economist, Esso Exploration Angola y www.gulfoilandgas.com.

Nota. Como ya advertimos para el número de campos en actividad no hemos completado la compulsa de fuentes, de ahí que los datos de nuestra columna no coincidan con las cifras de Petroleum

Economist. 
espléndida. Se han descubierto tres campos gigantes: Girasol; produciendo desde hace siete años, Saxe y Batuque, que apenas entraron en operación en agosto de 2008. ${ }^{6}$ El número de campos profundos frente a las costas de esta nación ya asciende a casi 30 y los que están en explotación son mayores que los de Brasil. Sólo entre junio de 2007 y marzo de 2008, en 10 meses, arrancaron ocho nuevos campos, ${ }^{7}$ a ellos se suman los dos gigantes ya mencionados, con lo que para septiembre de 2008 este país tendría 23 campos en desarrollo. Es indudable que este auge es impulsado por el actual pico de los precios.

No menos importantes son las innovaciones técnicas que se están desarrollando frente a este segmento de las costas africanas. En el campo Girasol se perforó un pozo horizontal, desviado 75 grados respecto de la vertical, en profundidades de 2,0002,750 metros del subsuelo. ${ }^{8}$

\section{Guinea Ecuatorial}

Los primeros tres campos en aguas profundas se perforaron en la segunda mitad de la década de los noventa y todos ellos iniciaron operaciones al año siguiente. ¿Cómo puede explicarse esta celeridad que no encontramos en ningún otro caso en esos años? Sólo contamos con informaciones incompletas y dispersas. El campo Zafiro fue descubierto por Exxon-Mobil en 1995, una zona cuyas fronteras marítimas se encontraban indefinidas, ¿acaso anticipándose a cualquier disputa, el gobierno de

6 Transcribimos el boletín que se emitió el 13 de agosto, desde Irving Texas: "Exxon Mobil Corporation announced today that its subsidiary, Esso Exploration Angola Limited (Esso Angola) has started production from the Saxi and Batuque fields" (www.businesswire.com/portal/site/ exxonmobil/index.jsp?ndmViewId=news_view...). Los interesados en conocer algunos comentarios adicionales sobre el conjunto del proyecto, al que pertenecen estos campos, (Kizomba C Project), pueden leer también el boletín: "Production Starts Up at Exxon's Saxi, Batuque Fields in Angola (www.rigzone.com/news/article).

7 La elaboración de nuestros cuadros presenta retrasos, es decir, no hemos podido concretar los datos en todos los casos; para esta información nos limitamos a citar las últimas cifras tomadas de Petroleum Economist: "Angola tiene ahora 21 campos en aguas profundas en producción, un incremento de ocho, solamente en los últimos 10 meses", En "Deep-water start-ups accelerate", http://www.petroleumeconomist.com/default.asp?page=14\&PubID=46\&ISS=24599\&SID=7 02930\&LS=EMS169187 Petroleum Economist Home /PE Magazine Archive /March 2008 / Article.

8 Véase por ejemplo este encabezado: "Campo Girasol: desplazamiento de la frontera de las aguas profundas", en un suplemento de una de las más importantes publicaciones texanas: Hart's E\&P, de mayo de 2002. 
Cuadro 3

Campos profundos en Guinea Ecuatorial

\begin{tabular}{|c|c|c|c|c|c|c|}
\hline Campo & $\begin{array}{c}\text { Fecha } \\
\text { de descubrimiento }\end{array}$ & $\begin{array}{l}\text { Fecha de inicio } \\
\text { de explotación }\end{array}$ & Tirante en metros & Compañía & Tipo de sistema & Situación actual \\
\hline 1. Zafiro & 1995 & 1996 & 600 & Mobil & $\mathrm{FP}$ & En producción \\
\hline 2. Topacio & 1996 & 1997 & 579 & Mobil & SS to FP & En producción \\
\hline 3. Ceiba & 1999 & 2000 & 700 & Amerada Hess & $\mathrm{FP}$ & En producción \\
\hline 4. Okume & 2001 & $----\cdot$ & 503 & Amerada Hess & -.------ & Virgen \\
\hline 5. Ceiba South & 2002 & $-\cdots-\cdot$ & 1,001 & Amerada Hess & -..---.- & Virgen \\
\hline 6. Ébano & 2002 & -.-- & 625 & Amerada Hess & ------.- & Virgen \\
\hline 7. Benita & 2007 & $-\cdots-$ & & Noble Energy Inc. & -.-----. & Virgen \\
\hline 8. Diega & 2008 & $-\cdots+$. & 660 & Noble Energy Inc. & -.--.--. & Virgen \\
\hline
\end{tabular}

Fuente: US DOE, Mobil Co., Amerada Hess Corporation y Noble Energy Inc.

Guinea aprobó su rápido desarrollo (a fast-track development) ? ${ }^{9}$ La Mobil Corporation (es decir Exxon) acondicionó un buque-tanque para reunir la producción de los pozos y exportarla.

Zafiro es un campo gigante, su producción inicial fue de 40 mil barriles diarios; en la actualidad aporta, según el DOE, poco menos de 250 mil barriles diarios.

Sobre el problema de las fronteras y el conflicto de límites que surgieron (antes o después de la decisión de explotar Zafiro y Topacio) sólo sabemos que en 2004 fue creada una zona de desarrollo conjunto, en tanto la Organización de las Naciones Unidas (ONU) dicte una resolución final. Quizás lo anterior retrasó el desarrollo de actividades frente a las costas de ese país. Hasta 2007 la Noble Energy, con sede en Houston, Texas, reemprendió la exploración. Se han descubierto dos nuevos campos, el más reciente apenas reportado en junio de $2008 .^{10}$

9 "The Zafiro Oil accumulation was discovered in March 1995. The field was declared commercial in october 1995. Approved by the government of Equatorial Guinea, Phase I is a fast-track development which include eight wells", en "Topacio-1 Well Extends Zafiro Field Offshore Equatorial Guinea”. Business Wire, March 21, 1966 (http://findarticles.com/p/articles/mi_ moEIN/IS_1996_March_21/ai_18113817). Véanse otras informaciones en el capítulo sobre Guinea en www.eia.doe.gov/emeu/cabs/Equatorial_Guinea/Oil.html.

10 Véase "Noble hits pay dirt in Ecuatorial Guinea (http://www.rigzone.com/news/article.asp?a id=62659) y Noble Energy, "Noble Energy announces test results from Benita oil appraisal Ecuatorial Guinea”, News Release, Houston, june 5, 2008 (www.nobleenergy.com). 
Congo

En este país también nos encontramos con campos marinos profundos que a pesar de haber sido descubiertos desde los años noventa apenas han iniciado operaciones. La francesa Total anunció el pasado 28 de abril de este 2008 que el complejo MohoBilondo comenzó a producir. ${ }^{11}$ La operadora destacó una reducción del ciclo entre el descubrimiento y el arranque de la producción. Explicó que aunque el campo fue descubierto en 1995 y transcurrieron 13 años de espera, "una vez firmado el contrato, sólo transcurrieron 33 meses para el diseño, construcción e instalación de los equipos, incluyendo el tendido de un oleoducto de 80 kilómetros de largo y 16 pulgadas de diámetro, que trasladará el crudo a la terminal de exportación en la costa. ${ }^{12} \mathrm{Se}$ espera que la producción se eleve hasta 90 mil barriles diarios, cifra exagerada si se considera el modesto volumen de reservas estimado para los dos campos, ahora unificados para su explotación, según Offshore, de 240 millones de barriles. ${ }^{13}$

Cuadro 4

Campos en aguas profundas del Congo

\begin{tabular}{|c|c|c|c|c|c|c|}
\hline Campo & $\begin{array}{c}\text { Fecha } \\
\text { de descubrimiento }\end{array}$ & $\begin{array}{l}\text { Fecha de inicio } \\
\text { de explotación }\end{array}$ & Tirante en metros & Compañía & Tipo de sistema & Situación actual \\
\hline 1. Moho & 1995 & 2008 & 800 & Total & ¿? & En producción \\
\hline 2. Bilondo & 1998 & 2008 & 546 & Total & ¿? & En producción \\
\hline 3. Lianzi & 2004 & ....... & 909 & Chevron & ........... & Virgen \\
\hline 4. Azurie & 2005 & -..-- & 1,376 & Murphy & -..--..- & Virgen \\
\hline
\end{tabular}

Fuente: Totalfina.

\section{Mauritania}

Éste es quizás el país que más recientemente ha sido incorporado a la carrera por la explotación de campos profundos. En 2001 la empresa australiana Woodside descubrió el primer campo, el Chinguetti, que en 2006 inició operaciones. Se trata de un yacimiento pequeño como puede verse por su modesta producción de 15 mil barriles

11 Total, "Moho-Bilondo Brought on stream Offshore the Republic of the Congo", april 28, 2008 (http://www.totalfina.net/) y "Chevron confirms startup.of Moho-Bilondo offshore Republic of the Congo"; Sn Ramón, Calif., April 29, 2008 (www.chevron.com).

12 Total, op. cit.

13 Offshore, 28 de abril, 2008 (www.offshore-mag.com).

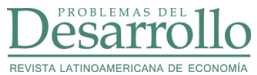


diarios. ${ }^{14}$ El Departamento de Energía de Estados Unidos ha anunciado que el campo Tiof arrancaría su producción en 2007, es decir este proyecto ya está retrasado. ${ }^{15}$

Cuadro 5

Campos de Mauritania

\begin{tabular}{lcccccc}
\hline Campo & $\begin{array}{c}\text { Fecha } \\
\text { de descubrimiento }\end{array}$ & $\begin{array}{c}\text { Fecha de inicio } \\
\text { de explotación }\end{array}$ & Tirante en metros & Compañía & Tipo de sistema & Situación actual \\
\hline 1. Chinguetti & 2001 & 2006 & 791 & Woodside & FP & En producción \\
2. Tiof & 2003 & $-\cdots---$ & 1,080 & Woodside & -.--- & Virgen \\
\hline
\end{tabular}

Fuente: US DOE.

\section{Costa de Marfil}

Igual que Mauritania este país apenas está iniciando perforaciones y explotación de sus campos profundos.

\section{Cuadro 6}

Campos de Costa de Marfil

\begin{tabular}{lcccccc}
\hline Campo & $\begin{array}{c}\text { Fecha } \\
\text { de descubrimiento }\end{array}$ & $\begin{array}{c}\text { Fecha de inicio } \\
\text { de explotación }\end{array}$ & Tirante en metros & Compañía & Tipo de sistema & Situación actual \\
\hline 1. Baobab & 2001 & 2005 & 1,000 & CNR International & FP & En producción \\
2. Acajou & 2003 & $-\cdots--$ & 930 & CNR International & $\cdots$ & Virgen \\
3. Espoir & $i ?$ & 2002 & 600 & CNR International & SS to onshore & En producción \\
\hline
\end{tabular}

* CNR: Canadian Natural Resources.

Fuente: www.rigzone.com y www.offshore-technology.com.

\section{Ghana}

Apenas el pasado mes de mayo de este 2008 la prensa petrolera mundial anunció el primer descubrimiento de un campo en aguas profundas de este país: el Mahogany, de la estadounidense Kosmos Energy.

\begin{tabular}{lccccccc}
\multicolumn{9}{c}{$\begin{array}{c}\text { Cuadro 7 } \\
\text { Campos de Ghana }\end{array}$} \\
\hline Campo & $\begin{array}{c}\text { Fecha } \\
\text { de descubrimiento }\end{array}$ & $\begin{array}{c}\text { Fecha de inicio } \\
\text { de explotación }\end{array}$ & Tirante en metros & Compañía & Tipo de sistema & Situación actual \\
\hline Mahogany & 2008 & $\cdots$ & $\cdots--$ & 1,320 & Kosmos Energy & $\cdots$ & Virgen \\
\hline
\end{tabular}

Fuente: Petroleum Economist.

14 DOE, IEA, Arab Mghreb Union, Country Analysis Briefs (www.eia.doe.gov).

15 Ibid. 
Australia

También en este país, con numerosos campos profundos descubiertos desde los años ochenta, la explotación apenas inicia en 2006, en el actual pico de los precios. Un estudio explica que algunos descubrimientos de gas se realizaron cuando la inexistencia de un mercado de gas licuado impedían su desarrollo. ${ }^{16}$ Enfield arranca operaciones en 2006 y Stybarrow en noviembre de 2007. ${ }^{17}$ Se encuentran en construcción los equipos para Eskdale ${ }^{18}$ y Gorgon. ${ }^{19}$

Cuadro 8

Campos profundos de Australia

\begin{tabular}{|c|c|c|c|c|c|c|}
\hline Campo & $\begin{array}{c}\text { Fecha } \\
\text { de descubrimiento }\end{array}$ & $\begin{array}{l}\text { Fecha de inicio } \\
\text { de explotación }\end{array}$ & Tirante en metros & Compañía & Tipo de sistema & Situación actual \\
\hline 1. Chrysaor & 1994 & --.-- & 806 & ¿? & - & Virgen \\
\hline 2. Dionysus & 1996 & ----- & 1,092 & $i ?$ & --.--.- & Virgen \\
\hline 3. Gorgon & 1997 & ----. & 920 & Chevron & ---.-- & Virgen \\
\hline 4. Enfield & 1999 & 2006 & 544 & BHP Billiton & $\mathrm{FP}$ & En producción \\
\hline 5. Jansz & 2000 & $-\cdots-\cdot$ & 1,321 & ¿? & - --.-- & Virgen \\
\hline 6. Laverda & 2000 & ---.- & 840 & ¿? & -----. & Virgen \\
\hline 7. Stybarrow & 2003 & 2007 & 825 & BHP Billiton & $\mathrm{FP}$ & En producción \\
\hline 8. Skiddaw & 2003 & -.--. & 780 & BHP Billiton & -----. & Virgen \\
\hline 9. Eskdale & 2004 & ...... & 822 & Woodside & -....... & Virgen \\
\hline 10. Pluto & 2005 & $-\cdots--$ & 976 & ¿? & --.--.- & Virgen \\
\hline
\end{tabular}

Fuente: Chevron, BHP Billiton y Offshore.

Indonesia

Igual, en Indonesia los primeros campos marinos fueron descubiertos desde los años noventa, pero el único campo en operación, el West Seno, tuvo que esperar hasta el pico de precios para iniciar operaciones.

16 "At the time, the available technology and the undeveloped LNG market the remote, deepwater gas accumulation uneconomic to develop", Marita Bradshaw, "Deepwater exploration offshore Australia picks up pace", Offshore, Tulsa, April oi, 2006

17 BHP Billiton Announces First Oil Production From Stybarrow Development off Western Australia”, 19 november 2007 (http://www.bhpbilliton.com/bb/investorsMedia/news/2007/.

18 BHP Billiton's Stybarrow Development off Western Australia Exceeding Early Forecast", January 15, 2008 (http://www.bhpbilliton.com/bb/investorsMedia/news/2008/.

19 Es un proyecto de gas natural licuado en la Isla Barrow, encabezado por Chevron, véase "Highlights of Operations", www.chevron.com/countries/australia/

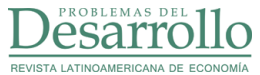


West Seno, en producción desde 2003, parece ser un campo gigante. En la actualidad explota más de más de 20 pozos y produce 40 mil barriles diarios. ${ }^{20}$ En este campo se construyó un sistema combinado de plataformas marinas con equipos de proceso en tierra. Comprende una plataforma de patas tensionadas (llamada TLP por sus siglas en inglés), dos unidades flotantes y ductos que conducen los hidrocarburos a tierra firme, a la terminal llamada Santan, en donde se procesan. Es un esquema que, como veremos, también se ha ensayado en Egipto y se estudia para el desarrollo de los campos profundos del proyecto Coatzacoalcos en México.

Cuadro 9

Campos profundos de Indonesia

\begin{tabular}{|c|c|c|c|c|c|c|}
\hline Campo & $\begin{array}{c}\text { Fecha } \\
\text { de descubrimiento }\end{array}$ & $\begin{array}{l}\text { Fecha de inicio } \\
\text { de explotación }\end{array}$ & Tirante en metros & Compañía & Tipo de sistema & Situación actual \\
\hline 1. Mera & 1997 & -..--.. & 520 & Chevron & -..---. & Virgen \\
\hline 2. West Seno & 1998 & 2003 & 953 & Chevron & TLP y FP to onshore & En producción \\
\hline 3. Aton & 1999 & $-\cdots+-\cdot$ & 1,150 & Chevron & ----- & Virgen \\
\hline 4. Janaka & 1999 & -..--.. & 1,316 & Chevron & ------.- & Virgen \\
\hline 5. Sadewa & 2003 & $-\cdots+-\cdot$ & 550 & Chevron & ------ & Virgen \\
\hline
\end{tabular}

*TLP: plataforma de patas tensionadas.

Fuente: Chevron.

Filipinas

Es un país importador neto, en el que los ingleses de Shell han incursionado en aguas profundas desde finales de los años ochenta. En 2001 se inició la producción de gas en el campo Malampaya. ${ }^{21}$

Cuadro 10

Campos profundos de Filipinas

\begin{tabular}{lcccccc}
\hline Campo & $\begin{array}{c}\text { Fecha } \\
\text { de descubrimiento }\end{array}$ & $\begin{array}{c}\text { Fechade inicio } \\
\text { de explotación }\end{array}$ & Tirante en metros & Compañía & Tipo de sistema & Situación actual \\
\hline 1. Camago & 1989 & $-\cdots--$ & 736 & Shell & $\ldots-\cdots--$ & Virgen \\
2. Malampaya-38 & 1992 & 2001 & 820 & Shell & Plat flexible & En producción \\
3. Malampaya (Rim) & 2000 & $-\cdots$ & 845 & Pnoc Energy & -.---- & Virgen \\
\hline
\end{tabular}

* PNOC: Philippine National Oil Company.

Fuente: Shell Philippines Exploration.

20 "Unocal. West Seno. Makassar Strait Deepwater Deveopment, Indonesia", http://www.offshore-technology.com/projects/west-seno/

21 Shell Philippines Exploration B.V. is operator of the US \$2 billion landmark Malampaya natural gas development project (http://www.shell.com). 
Malasia

También en este país la incursión a las aguas profundas apenas se inició. En 2002, la Murphy Oil Corporation, pequeña empresa estadounidense, realizó el primer descubrimiento, el campo Kikeh, anunciado como un "tremendo éxito". ${ }^{22}$ Un año después se descubrieron extensiones del mismo lugar. Iniciaron operaciones a finales de 2007, con una producción de 100 mil barriles diarios, lo que es un dato suficiente para reconocer a Kikeh como un nuevo campo gigante.

En este espacio se ha instalado una combinación de pozos controlados desde un equipo llamado Spar, con otros pozos submarinos. La Schlumberger lo califica como "único en su tipo, en el mundo". ${ }^{23}$ El proyecto comprende 20 pozos e incluye otros 20 pozos inyectores de agua.

Cuadro 11

Campo profundo en Malasia

\begin{tabular}{lcccccc}
\hline Campo & $\begin{array}{c}\text { Fecha } \\
\text { de descubrimiento }\end{array}$ & $\begin{array}{c}\text { Fecha de inicio } \\
\text { de explotación }\end{array}$ & Tirante en metros & Compañía & Tipo de sistema & Situación actual \\
\hline 1. Kikeh Sb & 2002 & $\ldots---$ & 1,330 & Murphy & $\cdots-\cdots--$ & En producción \\
2. Kikeh Kecil & 2003 & $\ldots-\cdots$ & 1,359 & Murphy & $\cdots-\cdots--$ & En producción \\
\hline
\end{tabular}

Fuente: Murphy Oil Corporation.

Japón

En este país las actividades en aguas profundas son recientes. Frente a las islas Hokkaido se descubrió el único campo; las pruebas de producción fueron exitosas pero las nuevas perforaciones para delimitar el yacimiento fracasaron. No obstante los contratiempos, el Japan Energy Group (JAPEX) afirma en un reporte que continuará la exploración con sísmica 3D y otras técnicas avanzadas. ${ }^{24}$

22 "Murphy achieved tremendous success at the Kikeh prospect located in 4400 feet of water" (http://www.murphyoilcorp.com/operations/expro/malaysia.aspx).

23 Ian Caulfield et al., "Manejo de proyectos de terminación de pozos marinos", Oilfield Review, Houston, verano 2007.

24 Japan Energy Development Co., Ltd, subsidiary of Japan Energy Corporation (http://www. jed.co.jp/activities/japan_sanriku.htm). 
Cuadro 12

Campo profundo de Japón

\begin{tabular}{lcccccc}
\hline Campo & $\begin{array}{c}\text { Fecha } \\
\text { de descubrimiento }\end{array}$ & $\begin{array}{c}\text { Fecha de inicio } \\
\text { de explotación }\end{array}$ & Tirante en metros & Compañía & Tipo de sistema & Situación actual \\
\hline 1. Sanriku 0ki & 2000 & $\ldots-\cdots$ & 857 & JAPEX & -.------ & Virgen \\
\hline
\end{tabular}

Fuente: Japan Energy Development.

India

India también inició actividades en sus aguas profundas en 2000. Ha descubierto cuatro campos, pero ninguno se encuentra en funcionamiento. Quizá porque ha sufrido problemas, como lo reporta la prensa petrolera internacional. En octubre de 2006, la plataforma Sagar Bhusan sufrió un accidente en el que se perdieron todos los equipos en el fondo del mar, tal vez ello retrasó sus programas. La petrolera estatal Oil and Natural Gas Corporation Limited (ONGC) anunció el descubrimiento de su campo Krishna como un "gran descubrimiento" de gas.

Cuadro 13

Campos profundos de India

\begin{tabular}{|c|c|c|c|c|c|c|}
\hline Campo & $\begin{array}{c}\text { Fecha } \\
\text { de descubrimiento }\end{array}$ & $\begin{array}{l}\text { Fecha de inicio } \\
\text { de explotación }\end{array}$ & Tirante en metros & Compañía & Tipo de sistema & Situación actual \\
\hline 1. Krishna & 2000 & $-\cdots-\cdot-$ & 844 & ONGC & -..--.- & Virgen \\
\hline 2. Annapurna & 2001 & $-\cdots--\cdot$ & 1,030 & ONGC & ------.- & Virgen \\
\hline 3. M Field & 2001 & $\cdots+.$. & 500 & ONGC & ........ & Virgen \\
\hline 4. D6 Field & 2002 & $-\cdots---$ & 900 & ¿? & -..--.. & Virgen \\
\hline
\end{tabular}

Fuente: www.ogj.com.

\section{Europa}

Noruega

Este país sólo tiene un campo en aguas profundas, el Ormen Lange, descubierto por Norsk Hidro en 1997, inició producción 10 años más tarde, en octubre de 2007, con Shell como operadora. Es un campo gasero gigante del que se espera una producción de 2,470 millones de pies cúbicos de gas diarios. Toda la producción está destinada a Reino Unido.

El Ormen Lange no se encuentra en el Mar del Norte sino en aguas más septentrionales del litoral de Noruega, cercanas al Circulo Polar. En temperaturas próximas 
al punto de congelamiento se forman hidratos en las corrientes de producción; para prevenirlos se instaló lo que se dice es "el sistema de anticongelantes más grande del mundo". ${ }^{25}$ Pero el desafío principal parece que fue la construcción del gasoducto que corre de la estación de proceso situada en Noruega y el litoral de Reino Unido. El ducto tiene 1,200 kilómetros de longitud y fue instalado a una profundidad de entre 850 y 1,100 metros. $^{26}$

Offshore Engineering concluye en un amplio reportaje que en Ormen Lange la instalación pasará a la historia de los proyectos offshore por este tipo de construcciones.

\begin{tabular}{lccccccc}
\multicolumn{9}{c}{$\begin{array}{c}\text { Cuadro 14 } \\
\text { Campo de Noruega }\end{array}$} \\
\hline Campo & $\begin{array}{c}\text { Fecha } \\
\text { de descubrimiento }\end{array}$ & $\begin{array}{c}\text { Fecha de inicio } \\
\text { de explotación }\end{array}$ & Tirante en metros & Compañía & Tipo de sistema & Situación actual \\
\hline Ormen lange & 1997 & 2007 & 888 & Shell & SS to onshore & En producción \\
\hline
\end{tabular}

Fuente: www.hydro.com

\section{Reino Unido}

Desde la primera mitad de los noventa este país descubrió en sus aguas profundas sus primeros campos en áreas del margen del Atlántico. Después de casi 15 años ninguno de ellos ha producido. Nuestra pesquisa nos permite concluir que en el Mar del Norte no se han descubierto campos en profundidades de 500 metros o más, los únicos tres campos profundos se ubican al noroeste de Escocia, cerca de las islas Faroes.

Cuadro 15

Campos profundos de Reino Unido

\begin{tabular}{|c|c|c|c|c|c|c|}
\hline Campo & $\begin{array}{c}\text { Fecha } \\
\text { de descubrimiento }\end{array}$ & $\begin{array}{l}\text { Fecha de inicio } \\
\text { de explotación }\end{array}$ & Tirante en metros & Compañía & Tipo de sistema & Situación actual \\
\hline 1. Cuillin Central & 1994 & -.--.. & 510 & $B P$ & -..--.- & Virgen \\
\hline 2. Cuillin South & 1994 & -.-- & 530 & $B P$ & -..-- & Virgen \\
\hline 3. Alligin & 1995 & ----- & 514 & $B P$ & -.--- & Virgen \\
\hline
\end{tabular}

Fuente: British Petroleum.

25 "Ormen Lange, North Sea Northern, Norway (www.offshore-technology.com/projects/ormen/.

26 Otra vez se dice que es "el ducto submarino más grande del mundo" (Boletín de prensa del Norwegian Ministry of Foreign Affairs, 24 octubre 2006). 
La British Petroleum ha incluido uno de ellos en la lista de proyectos que pretende realizar; sin mucho entusiasmo señala que se encuentran "entre los proyectos bajo consideración". ${ }^{27}$

Albania

Inmediatamente después del derrumbe del socialismo este país abrió su sector del Mar Adriático a las licitaciones internacionales. En 1993 una asociación de Chevron y la italiana AGIP perforó un pozo profundo reportado con manifestaciones de hidrocarburos. El propio gobierno ha publicado que el descubrimiento no es comercial, quizá nunca se explote..$^{28}$

\begin{tabular}{|c|c|c|c|c|c|c|}
\hline \multicolumn{7}{|c|}{$\begin{array}{c}\text { Cuadro } 16 \\
\text { Campo de Albania }\end{array}$} \\
\hline Campo & $\begin{array}{c}\text { Fecha } \\
\text { de descubrimiento }\end{array}$ & $\begin{array}{l}\text { Fecha de inicio } \\
\text { de explotación }\end{array}$ & Tirante en metros & Compañía & Tipo de sistema & Situación actua \\
\hline 1. A4-IX & 1997 & -..-- & 700 & AGIP (Italia) & -..---. & Virgen \\
\hline
\end{tabular}

Fuente: AGIP (www.agippetroli.it/index.html).

Italia

Muy temprano la Agencia Generale Italiana Petroli (AGIP) perforó, asociada con Chevron, un pozo profundo, el "Aquila". ${ }^{29}$ Considerado en un inicio como "poco rentable" por su ubicación remota y escasa reserva, unos 20 millones de barriles, fue desarrollado con perforaciones horizontales, rama en la que los italianos han logrado importantes desarrollos submarinos. ${ }^{30}$

Cuadro 17

Campo de Italia

\begin{tabular}{ccccccc}
\hline Campo & $\begin{array}{c}\text { Fecha } \\
\text { de descubrimiento }\end{array}$ & $\begin{array}{c}\text { Fecha de inicio } \\
\text { de explotación }\end{array}$ & Tirante en metros & Compañía & Tipo de sistema & Situación actual \\
\hline 1. Aquila & $\dot{\imath}$ ? & 1998 & 850 & Agip (Italia) & FP & En producción \\
\hline
\end{tabular}

Fuente: Offshore, Houston, Texas, 22 de junio de 1999.

27 Véase D. Turner, BP North Sea Subsea Manager, The Role \& Importance of Subsea", $27^{\text {th }}$ June 2006 (http://www.subseauk.org/documents/BP\%20-20onenortheastbrrakfast.pdf).

28 Véase "Exploration and Production History in Albania" (http://www.akbn.gov.al/files/exploration_production_history.pdf).

29 Algunas fuentes aseguran que en los ochenta, otras que en 1993.

30 ENI ha propagandizado que en asociación con Schlumberger perforó el pozo horizontal más profundo del mundo en Villafortuna Trecate en 21066 pies de profundidad y un ángulo de $89.6^{\circ}$ (véase World Oil, agosto de 2001).

Vol. 39, núm. 155, octubre-diciembre / 2008

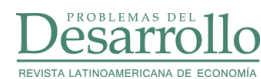


Israel

En este país se han descubierto tres campos profundos. Ninguno se encuentra en explotación. Recientemente la estadounidense Noble Energy descubrió el campo que parece ser el más importante de esta nación, el Mari-B, con localizaciones en someras y profundas. ${ }^{31}$

Cuadro 18

Campos profundos de Israel

\begin{tabular}{|c|c|c|c|c|c|c|}
\hline Campo & $\begin{array}{c}\text { Fecha } \\
\text { de descubrimiento }\end{array}$ & $\begin{array}{l}\text { Fecha de inicio } \\
\text { de explotación }\end{array}$ & Tirante en metros & Compañía & Tipo de sistema & Situación actual \\
\hline 1. Noa & 1999 & -.---.- & 779 & Samedan & -.---.-.- & Virgen \\
\hline 2. Mari-B & 2007 & -.---- & 1,646 & Noble Energy Inc. & -.---.-. & Virgen \\
\hline 3. Tamar & 2007 & --.--- & 1,620 & Noble Energy Inc. & ------.- & Virgen \\
\hline
\end{tabular}

Fuente: Noble Energy Inc.

África Mediterráneo

Egipto

Los primeros campos profundos se perforaron en 1998, pero su desarrollo, como en múltiples casos aquí examinados, sólo se inició hasta el periodo actual de precios al alza.

Como ya adelantamos, en este país se han construido sistemas de explotación que combinan las instalaciones de extracción costa afuera con equipos de proceso en tierra, además, incorporando campos profundos y campos en aguas someras. Así, los campos Sienna, Simian, Scarab, en profundas y otros en someras como Sapphire, pertenecen a un proyecto de desarrollo conjunto, muy cercano al Delta del Nilo; la distancia entre los campos y las instalaciones de proceso de gas, en tierra, cerca de

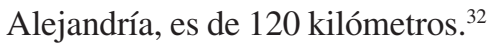

31 La empresa asegura que contiene reservas de un billón de pies cúbicos de gas, Oil \& Gas Journal, febrero 18 de 2002 y "Noble affiliates announces more success offshore Israel”, The Journal Record, Oklahoma City, May 18, 2000 (http://findarticles.com/p/articles/mi_9n4182/ is_20000518/ai-n101137886).

32 Véase "Deepstar gas development off Egypt overcomes challenges", Oil \& Gas Journal, 10 febrero 2003. La administración general del proyecto fue entegada a Bechtel, igual que ocurrió en el caso del proyecto de inyección de gas nitrógeno a Canterell en México ("Innovative subsea development solution for deepwatwer Egyptian field", Pipeline \& Gas Journal, august 2002, http://findarticles.com/p/articles/mi_m3251/is_8_229/ai-N25053380. 
Cuadro 19

Campos profundos en Egipto

\begin{tabular}{|c|c|c|c|c|c|c|}
\hline Campo & $\begin{array}{c}\text { Fecha } \\
\text { de descubrimiento }\end{array}$ & $\begin{array}{l}\text { Fecha de inicio } \\
\text { de explotación }\end{array}$ & $\begin{array}{l}\text { Tirante } \\
\text { en metros }\end{array}$ & Compañía & Tipo de sistema & Situación actual \\
\hline 1. Saffron & 1998 & 2003 & 610 & Burullus Gas Co. & SS to onshore facilities & En producción \\
\hline 2. Scarab & 1998 & 2003 & 650 & Burullus Gas Co. & SS to onshore facilities & En producción \\
\hline 3. Simian & 1999 & 2005 & 790 & Burullus Gas Co. & SS to onshore facilities & En producción \\
\hline 4. Serpent & 1999 & $-\cdots---$ & 600 & Burullus Gas Co. & ...-..- & Virgen \\
\hline 5. Sienna & 2000 & 2005 & 985 & Burullus Gas Co. & SS to FP & En producción \\
\hline 6. Saurus & 2001 & 2004 & 630 & Burullus Gas Co. & -..--- & Virgen \\
\hline 7. Sequoia & 2002 & $\cdots---$ & 600 & Burullus Gas Co. & -.----- & Virgen \\
\hline 8. Solar & 2002 & $-\cdots--$ & 700 & Burullus Gas Co. & -..--.-. & Virgen \\
\hline 9. El King & 2002 & -..--- & 720 & Hess Corp. & -.------ & Virgen \\
\hline 10. El Max & 2002 & -.--- & 945 & Hess Corp. & ...--.-- & Virgen \\
\hline
\end{tabular}

Fuente: Offshore y Petroleum Economist, diversos números.

\section{América Latina}

Brasil

Este país es un caso similar al de Estados Unidos, acicateado por la escasez se adelantó una década en las perforaciones en aguas profundas y logró iniciar la explotación de sus campos desde comienzos de los años noventa, como puede verse en el siguiente cuadro, con el caso de Marlim.

El campo más profundo en explotación es Roncador en poco más de 1,850 metros de profundidad. Con los recientes descubrimientos realizados en 2007 y 2008, ahora esa nación tendrá que explotar campos en tirantes de más de 2,000 metros.

En abril y noviembre de 2007 y enero de 2008 en Brasil se realizaron tres descubrimientos que conmovieron al mundo: Tupi, Sugar y Júpiter. Destacamos un componente tecnológico en los hallazgos: los tres campos se encuentran en una antigua cuenca en la que ya se habían perforado unos 100 pozos, es decir, se trata de una "reentrada", con herramientas de sísmica con mejor resolución, que permitió mejorar las imágenes superando los problemas de los cuerpos salinos en el subsuelo. Los funcionarios de PETROBRAS esperan que entre cinco y siete años más Brasil se encontrará explotando campos en más de 2,230 metros de profundidad de agua, lo que hoy sólo ocurre en Estados Unidos.

Existen numerosas informaciones sobre el potencial de los yacimientos mencionados; la prensa afirma de la existencia de un potencial entre cinco y ocho mil millones de barriles, desde luego aún no se cuenta con estadísticas oficiales.

Vol. 39, núm. 155, octubre-diciembre / 2008

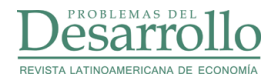


FABIO BARBOSA CANO

120

Cuadro 20

Campos profundos de Brasil

\begin{tabular}{|c|c|c|c|c|c|c|}
\hline Campo & $\begin{array}{c}\text { Fecha } \\
\text { de descubrimiento }\end{array}$ & $\begin{array}{l}\text { Fecha de inicio } \\
\text { de explotación }\end{array}$ & Tirante en metros & Compañía & Tipo de sistema & Situación actual \\
\hline 1. Marlim & 1985 & 1991 & 835 & PETROBRAS & $\mathrm{FP}$ & En producción \\
\hline 2. Frade (Area 366) & 1986 & $-\cdots--$ & 1,141 & CHEVRON & $\cdots--$ & Virgen \\
\hline 3. Marlim South & 1987 & 2001 & 1,080 & PETROBRAS & $\mathrm{FP}$ & En producción \\
\hline 4. Marlim module 1 & 1987 & 1994 & 1,027 & PETROBRAS & SS to FP & En producción \\
\hline 5. Voador South & 1987 & 1998 & 611 & PETROBRAS & FP & En producción \\
\hline 6. Barracuda & 1989 & 2004 & 843 & PETROBRAS & FP & En producción \\
\hline 7. Voador North & 1989 & 1998 & 563 & PETROBRAS & SS to FP & En producción \\
\hline 8. Area Do 1-425 & 1990 & 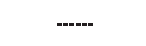 & 925 & PETROBRAS & $\cdots--$ & Virgen \\
\hline 9. Bijupira & 1990 & 2003 & 735 & Shell & $\mathrm{FP}$ & En producción \\
\hline 10. Salema & 1990 & 2003 & 550 & Shell & SS to FP & En producción \\
\hline 11. Espadarte 409 & 1993 & $-\cdots-\cdot$ & 1,350 & PETROBRAS & $-\cdots--$ & Virgen \\
\hline 12. Marlim West & 1993 & 1999 & 700 & PETROBRAS & SS to FP & En producción \\
\hline 13. Albacora Leste & 1994 & 2006 & 1,240 & PETROBRAS & SS to FP & En producción \\
\hline 14. Albacora Pilot & 1994 & 1998 & 1,109 & PETROBRAS & SS to FP & En producción \\
\hline 15. Caratinga & 1994 & 2005 & 922 & PETROBRAS & $\mathrm{FP}$ & En producción \\
\hline 16. Espadarte 499 & 1994 & 2000 & 940 & PETROBRAS & $\mathrm{FP}$ & En producción \\
\hline 17. Marlim Pilot & 1994 & 1998 & 1,250 & PETROBRAS & SS to FP & En producción \\
\hline 18. Marlim Leste & 1994 & $-\cdots--\cdot$ & 1,080 & PETROBRAS & -.---. & Virgen \\
\hline 19. Frade 511 & 1996 & $\cdots$ & 980 & Chevron & $\cdots--$ & Virgen \\
\hline 20. Area 509-A & 1996 & $-\cdots-$. & 962 & PETROBRAS & $-\cdots--$ & Virgen \\
\hline 21. Marimba Leste & 1996 & 2001 & 700 & PETROBRAS & SS to FP & En producción \\
\hline 22. Roncador & 1996 & 2000 & 1,853 & PETROBRAS & $\mathrm{FP}$ & En producción \\
\hline 23. Brazil-10 & 2000 & $-\cdots-.-$ & 1,654 & Shell & $-\cdots---$ & Virgen \\
\hline 24. Brazil 142 & 2001 & $-\cdots-\cdot$ & 531 & PETROBRAS & $\cdots--$ & Virgen \\
\hline 25. Marimba Leste & 2001 & $-\cdots--\cdot$ & 711 & PETROBRAS & $\cdots--$ & Virgen \\
\hline 26. Jubarte BC-60 & 2001 & 2008 & 1,246 & PETROBRAS & $\mathrm{FP}$ & En producción \\
\hline 27. Jubarte Main & 2001 & $-\cdots-$. & 1,246 & PETROBRAS & $\cdots--$ & Virgen \\
\hline 28. Brazil SPS 36 & 2003 & $\cdots$ & 1,000 & PETROBRAS & $\cdots-$ & Virgen \\
\hline 29. Golfinho & 2003 & 2006 & 1,350 & PETROBRAS & $\mathrm{FP}$ & En producción \\
\hline 30. Golfinho Pilot & 2003 & 2006 & 1,300 & PETROBRAS & $\mathrm{FP}$ & En producción \\
\hline 31. Golfinho Phase 1 & 2003 & $\cdots+.$. & 1,374 & PETROBRAS & $\cdots+.$. & Virgen \\
\hline 32. Marlim Leste 9 & 2003 & $-\cdots--$. & 962 & PETROBRAS & 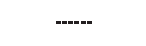 & Virgen \\
\hline 33. Piranema & 2003 & $\cdots$ & 1,152 & PETROBRAS & $\cdots--$ & Virgen \\
\hline 34. Canapu & 2005 & $-\cdots-$ & 1,608 & PETROBRAS & $-\cdots--$ & Virgen \\
\hline 35. Golfinho Northwest & 2006 & 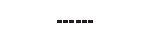 & 1,300 & PETROBRAS & $\cdots+-$ & Virgen \\
\hline 36. Tupi & 2007 & $-\cdots-$. & 2,231 & PETROBRAS & -.---. & Virgen \\
\hline 37. Sugar Loaf & 2007 & -.-.- & ¿? & PETROBRAS & -..-- & Virgen \\
\hline 38. Júpiter & 2008 & ....... & ¿? & PETROBRAS & ....... & Virgen \\
\hline
\end{tabular}

Fuente: Petroleo Brasileiro, S.A., para los últimos tres campos: www.rigzone.com y World Oil.

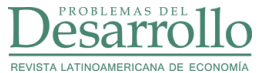

Vol. 39, núm. 155, octubre-diciembre / 2008 
México

PEMEX descubrió entre 2004 y 2007 cinco campos en profundidades mayores de 500 metros.

La Unidad Especializada en Aguas Profundas de PEMEX había elaborado un programa planteando avances graduales hacía el Golfo de México profundo; se proponía perforar 11 pozos exploratorios entre 2002 y 2007, además de investigaciones con instrumentos modernos de sísmica. ${ }^{33}$ Pero al coincidir con la inesperada elevación de los precios, la Secretaría de Energía reformuló su programa y desde finales de 2006 PEMEX se propuso perforar 47 pozos profundos sólo entre 2007 y 2012. ${ }^{34}$ Aduce que sin el aporte de nuevos campos en el área mencionada será inevitable una caída aún más pronunciada de la producción mexicana. ${ }^{35}$

En marzo de 2008 PEMEX creó un nuevo activo para la explotación de los primeros campos ya descubiertos en el proyecto Coatzacoalcos, fundamentalmente Lakach, Noxal y Lalail. En esta área ya se han descubierto reservas totales de 2.4 billones de pies cúbicos diarios. Los primeros conceptos de ingeniería de desarrollo que se han publicado sugieren un esquema similar al que hemos descrito en los casos de los campos del Delta del Nilo o el Kikeh de Malasia: un spar o una plataforma de patas tensionadas (TLP), para reunir la producción de los campos, en combinaciónn con equipos de separación, bombeo y proceso en tierra; en el caso del proyecto Coatzacoalcos, se ubicarían en Ciudad Lerdo de Tejada, Veracruz.

Cuadro 21

Campos profundos de México

\begin{tabular}{|c|c|c|c|c|c|c|}
\hline$\overline{C a m p o}$ & $\begin{array}{c}\text { Fecha } \\
\text { de descubrimiento }\end{array}$ & $\begin{array}{l}\text { Fecha de inicio } \\
\text { de explotación }\end{array}$ & Tirante en metros & Compañía & Tipo de sistema & Situación actual \\
\hline 1. Nab & 2004 & $-\cdots---$ & 679 & PEMEX & $-\cdots-\cdots$ & Virgen \\
\hline 2. Noxal & 2006 & -.--- & 936 & PEMEX & -..--.- & Virgen \\
\hline 3. Lakach & 2006 & $-\cdots---$ & 988 & PEMEX & -..--.- & Virgen \\
\hline 4. Lalail & 2007 & $-\cdots---$ & 806 & PEMEX & $-\cdots---$ & Virgen \\
\hline 5. Tamil & 2008 & -..-- & 660 & PEMEX & 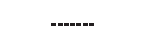 & Virgen \\
\hline
\end{tabular}

Fuente: Petróleos Mexicanos.

33 Guillermo Pérez Cruz et al., "Exploring the Mexican Sector of the Deepwater Gulf of Mexico", Paper presented at AAPG International Conference, Cancun, Mexico, october 24-27, 2004.

34 PEMEX Exploración y Producción, Documento representativo y Anuario Estadístico 2006. Región Norte, México, PEMEX, 2006.

35 Secretaría de Energía, Diagnóstico: situación de PEMEX, México, SENER, 2008 (www.pemex. com). 
El siguiente cuadro resume el conjunto de la estadística recopilada.

Cuadro 22

Campos descubiertos y en explotación en el mundo

\begin{tabular}{|c|c|c|c|c|}
\hline País & Descubiertos & En explotación & $\begin{array}{c}\text { Iniciaron operaciones } \\
\text { entre } 2003 \text { y } 2008\end{array}$ & Virgenes \\
\hline Nigeria & 11 & 4 & 4 & 7 \\
\hline Angola & 30 & 23 & 22 & 7 \\
\hline Ginea Ecuatorial & 8 & 3 & -.---.- & 5 \\
\hline Congo & 4 & 2 & 2 & 2 \\
\hline Mauritania & 2 & 1 & 1 & 1 \\
\hline Costa de Marfil & 3 & 2 & 1 & 1 \\
\hline Ghana & 1 & $-\cdots--\cdot$ & -..--- & 1 \\
\hline Total África & 59 & 35 & 30 & 24 \\
\hline Australia & 10 & 2 & 2 & 8 \\
\hline Indonesia & 5 & 1 & 1 & 4 \\
\hline Filipinas & 3 & 1 & -.---- & 2 \\
\hline Malasia & 2 & 2 & 2 & $-\cdots-\cdot-$ \\
\hline Total Australia y sureste de Asia & 20 & 6 & 5 & 14 \\
\hline Japón & 1 & $-\cdots-\cdots$ & -.---- & 1 \\
\hline India & 4 & 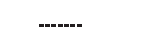 & 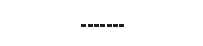 & 4 \\
\hline Noruega & 1 & 1 & 1 & $---\cdot-$ \\
\hline Reino Unido & 3 & -..--.. & -..--- & 3 \\
\hline Albania & 1 & 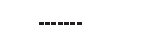 & -..--.- & 1 \\
\hline Italia & 1 & 1 & -..--- & $-\cdots--\cdot$ \\
\hline |srael & 3 & 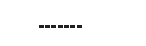 & ‥--- & 3 \\
\hline Egipto & 10 & 4 & 4 & 6 \\
\hline Brasil & 38 & 19 & 7 & 19 \\
\hline México & 5 & $-\ldots-\ldots$ & -..--- & 5 \\
\hline Total (menos EU) & 146 & 66 & 47 & 80 \\
\hline EU & 181 & 115 & 54 & 66 \\
\hline Total mundial & 327 & 181 & 101 & 146 \\
\hline
\end{tabular}

Fuente: Elaborado con los datos anteriores.

\section{Conclusiones}

1) La investigación ha revelado que antes del actual ascenso de precios sólo existían 16 campos profundos operando en el mundo, excepto Estados Unidos. Diez en Brasil, cuatro distribuidos en la cuenca del Níger en África, uno en Italia y otro en Filipinas. Es decir, la explotación petrolera en mares profundos, con excepción de Estados Unidos y Brasil, era una rareza. Hoy tenemos cerca de setenta campos en 
explotación, la gran mayoría inició operaciones entre 2003 y 2008. El gran impulso a la explotación en aguas profundas es una consecuencia del actual ascenso de los precios del crudo.

Los 16 campos que comenzaron a ser explotados antes del ascenso de precios se ubican en países que han agotado su dotación geológica o con escasas o nulas posibilidades en tierra, como Estados Unidos, Brasil y algunos países del Mediterráneo europeo.

2) En el periodo actual, caracterizado por la intensificación de la exploración, la febril construcción de plataformas y equipos para explotar los campos ya descubiertos y la investigación para apurar la producción, proceso que hemos llamado "una carrera contra el tiempo", destacan algunos segmentos: a la costa occidental de África y Brasil, que junto con el Golfo de México, se le denomina "el triángulo de oro". El occidente africano parece ser el escenario del mayor dinamismo de los descubrimientos y las nuevas explotaciones. Entre 2003 y la fecha actual en esa región han arrancado operaciones más de 30 campos en aguas profundas, es decir, han iniciado operaciones casi cuatro campos cada año. ${ }^{36}$ Sólo en Angola existen ahora 30 campos profundos, un número menor, pero puede ser comparado con Brasil, que tiene 38 campos profundos.

3) Por el elevado número de campos descubiertos y todavía sin producir, puede afirmarse que las actividades petroleras en aguas profundas apenas se encuentran en las primeras etapas de desarrollo y que esta carrera continuará. El problema del repunte de precios es un asunto específico que requiere un análisis especial, no sabemos en qué medida intervienen en él los problemas de oferta y los de orden político, lo que es un hecho es que está surgiendo una nueva rama, o especialidad en la industria petrolera.

4) Con el crecimiento de las perforaciones profundas, la investigación y el desarrollo tecnológico, que siempre han sido fundamentales, cobran un papel aún más importante. Hasta ahora en ningún país del mundo, fuera de Estados Unidos, existen

36 En los incisos correspondientes a cada país no pudimos desagregar los datos de producción; en un esfuerzo de presentar un panorama, señalemos que la extracción en el conjunto de los campos profundos de África en 2007 fue de 4.5 millones de barriles diarios, la Consultoría Douglas-Westwood estima un incremento de $80 \%$, es decir a 8.1 millones de barriles diarios para 2012; para ello, según la misma fuente, será necesario que las inversiones en infraestructura y equipos de los diversos países alcancen los 24.6 miles de millones de dólares, también para 2012 (Adrian Jonh \& Georgie MacFarlan Douglas-Westwood Ltd, “Out of Africa...”, ya citado). 
campos marinos en explotación en más de 1,000 metros de profundidad, con dos excepciones: Roncador, de PETROBRAS, en 1853 metros del "espejo del agua", como dicen ellos, y Rosa, de Total, en 1,405 metros, en Angola. Se insiste: hasta hoy sólo en las aguas territoriales de Estados Unidos, en el Golfo de México, existen campos en explotación de más de 2,000 metros. En el futuro este tipo de experiencias se presentarán en otros países; Brasil ya las está anunciando para 2013 o 2015, afirman sus funcionarios.

5) Nuestra investigación, aunque no pudo profundizar, por lo menos ha planteado el problema del acortamiento del ciclo entre el descubrimiento y el arranque de la producción de los campos profundos. En el caso de campos que fueron descubiertos hace más de una década y no fueron desarrollados, probablemente porque los precios vigentes no cubrían costos, el acortamiento se mide, como vimos en el caso de Moho-Bilondo, de la francesa Total en el Congo, entre la firma del contrato de construcción y la puesta en marcha del proyecto; en el ejemplo citado el nuevo ciclo comprendió 33 meses. El caso de los campos Zafiro y Topacio, de Mobil Co. en Guinea Ecuatorial, es sorprendente y debería ser objeto de un estudio específico. Nos permitiremos dejar planteadas nuestras dudas: ¿se habrán dañado los yacimientos?, ¿se habrá sacrificado la eficiencia de la recuperación?, ¿podrían ocurrir en México estas situaciones, si PEMEX se empeña en acelerar la explotación de sus campos profundos, como los que mencionamos del Proyecto Coatzacoalcos?

6) En el momento presente, más de la mitad de los campos descubiertos en el mundo, fuera de Estados Unidos, está en espera de que se construyan las plataformas y equipos para su explotación. Si a la extensa lista se agrega EU, tenemos más de 140 campos demandando infraestructura para su desarrollo. Desde luego lo anterior implica una gran demanda para las industrias de construcción de plataformas, tubería y otros artefactos electrónicos, así como de personal calificado para esta nueva especialidad que está emergiendo en la industria. En tales condiciones las compañías proveedoras han elevado precios y exigido mejorar los términos de sus contratos. Es un tema que no abordamos aquí, por falta de espacio, pero del que nos hemos ocupado en otros lugares. ${ }^{37}$

37 Véase "El programa de exploración y perforaciones en aguas profundas de PEMEX en el Golfo de México se encuentra paralizado" en http://jornada.unam.mx/ultimas/especiales/casa -lamm/mexico-y-el-mundo-actual. 
7) Finalmente, sería un error que México se precipite hacía el Golfo de México profundo para competir por posiciones en el mercado de crudos. Este país no tiene por qué involucrarse en la carrera contra el tiempo hacia las aguas profundas, porque, como numerosos analistas han explicado, aún tiene muchas oportunidades en aguas someras y en tierra; las disminuciones de su renta petrolera se están compensando con los mayores ingresos por el alza de precios.

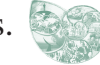

\section{Bibliografía}

Adrian John \& Georgie MacFarlan DouglasWestwood Ltd, "Out of Africa. Deepwater Prospects $\$ 24.6 \mathrm{~B}$ by 2012", Maritime Reporter \& Engineering News, november 2007.

Akin Iwayemi, "Africa and the Geopolitics of World Oil \&Gas: perspectives, challenges and opportunities", Ibadan, University of Ibadan, Paper presented at the 27 th USAEE/ IAEE North American Conference: Developing and Delivering Affordable Energy in the $21^{\text {st }}$ Century, Houston, September 17, 2007.

Berman, Arthur, "Three super giant fields discovered offshore Brazil", World Oil, vol. 229, núm. 2, February 2008.

Secretaría de Energía, Diagnóstico: situación de Pemex, México, SENER, 2008

Boletines de prensa de las siguientes instituciones y empresas:

Chevron (www.chevron.com)

Japan Energy Corporation (http://www.jed. co.jp/)

Vol. 39, núm. 155, octubre-diciembre / 2008
Murphy Oil Corporation (www.murphyoilcorp. $\mathrm{com} /$ )

Noble Energy Inc., Annual Analyst Meeting, (http, www.noble energyinc.com)

Petroleo Brasileiro, (www.petrobras.com.br/).

Petróleos Mexicanos (www.pemex.com)

Totalfina (http://www.total.com)

Publicaciones periódicas:

DOE, EIA, Country Analysis Briefs (www.eia. doe.gov).

Hart's E\&P, Houston.

Offshore, Houston.

Offshore-Engeenering, Tulsa, Ok.

Oil \& Gas Journal, Houston, Tx.

Petroleum Economist, Londres.

Rigzone (www.rigzone.com)

Oilfield Review, Publicación trimestral de Schlumberger Exploration and Production, Houston. 